\title{
Fusaricidins in Paenibacillus polymyxa A21 and their antagonistic activity against Botrytis cinerea on tomato
}

\author{
Weicheng LIU ${ }^{1 *}$, Xiaoli WU ${ }^{2 *}$, Xuelian BAI ${ }^{1}$, Hong ZHANG ${ }^{1}$, Dan DONG ${ }^{1}$, Taotao ZHANG ${ }^{1}$, Huiling WU (凶) ${ }^{1}$ \\ 1 Institute of Plant and Environment Protection, Beijing Academy of Agriculture and Forestry Sciences, Beijing 100097, China \\ 2 Editorial Department of Chinese Science Abstracts, Science and Technology Review Publishing House, Beijing 100081, China
}

\begin{abstract}
Four kinds of antifungal compounds from an extract of Paenibacillus polymyxa A21 with molecular masses of 883.56, 897.59, 947.55, and 961.58 Da were characterized as the members of fusaricidin-type of antibiotics according to LC-MS analysis. Fusaricidins isolated from culture filtrate displayed high antagonistic activity against several plant fungal pathogens, especially Botrytis cinerea, the causal agent of gray mold. The fusaricidins biosynthetic gene cluster (BGC) from A21 was cloned by PCR and comparative cluster analysis revealed that gene fusTE, the 3' boundary of the fusaricidin $\mathrm{BGC}$ in strain PKB1, was not present in fusaricidin BGC of $\mathrm{A} 21$, indicating that fusTE is not necessary for fusaricidin synthesis. Fusaricidin extract from A21 significantly reduced gray mold disease incidence and severity on tomato. The mRNA levels for three pathogenesis-related proteins (PRs) revealed that treatment of tomato leaves with fusaricidin extract induced the expression of PR genes to different levels, suggesting that one reason for the reduction of gray mold infection by fusaricidin is induction of PR proteins, which lead to increased resistance to pathogens. This is the first report of the application of fusaricidins to control tomato gray mold and the comparative cluster analysis provides important molecular basis for research on fusaricidin biosynthesis.
\end{abstract}

Keywords antifungal activity, biosynthetic gene cluster, Botrytis cinerea, fusaricidin, Paenibacillus polymyxa

\section{Introduction}

Paenibacillus polymyxa formerly Bacillus polymyxa ${ }^{[1]}$, is a plant growth-promoting rhizobacteria (PGPR) ${ }^{[2]}$ that can produce antimicrobial compounds, including hydrolytic

Received January 22, 2017; accepted May 8, 2017

Correspondence: wuhuiling925@126.com

${ }^{\text {*}}$ These authors contribute equally to the work enzymes and antibiotics, to control plant pathogens ${ }^{[3,4]}$. The antibiotics produced by $P$. polymyxa are known to include two types. One type, consisting of polymyxin, polypeptin, gavaserin, saltavalin, and jolipeptin, is only active against bacteria ${ }^{[5-8]}$, and the other type is made up of gatavalin and fusaricidin ${ }^{[9-12]}$.

Fusaricidins are a family of peptide antibiotics consisting of a group of cyclic depsipeptides with an unusual 15guanidino-3-hydroxypentadecanoic acid moiety bound to a free amino group ${ }^{[11]}$, and they have been identified as the potential antifungal agents produced by $P$. polymyxa $a^{[10]}$. So far, 12 fusaricidin analogs have been reported from multiple strains of Paenibacillus spp., including LI-F03a (fusaricidin C), LI-F03b (fusaricidin D), LI-F04a (fusaricidin A), LI-F04b (fusaricidin B), as well as a and b analogs of LI-F05 to LI-F08 ${ }^{[13]}$. The fusaricidin biosynthetic gene cluster (BGC) has been characterized in $P$. polymyxa strain PKB1. It spans $32.4 \mathrm{~kb}$ and contains 7 ORFs (fusB, fusC, fusD, fusE, fusF, fus $G$ and fusTE) and a large ORF (fusA) of about $23.7 \mathrm{~kb}$, encoding a six-module nonribosomal peptide synthetases involved in fusaricidin production. No genes were found for regulation, resistance or transport in this cluster ${ }^{[14]}$. Different fusaricidin-types and antimicrobial activities have been reported in the Paenibacillus spp. For example, fusaricidins A, B, C and D produced by strain SQR-2 $1^{[15]}$ is active against Fusarium oxysporum f. sp. nevium, while the LI-F type antibiotics LI-F03, LIF04, LI-F05, LI-F06, LI-F07 and LI-F08 produced by $P$. polymyxa $\mathrm{L}-1129$ and $P$. polymyxa I have excellent activity against Staphylococcus aureus ${ }^{[16,17]}$.

Botrytis cinerea is a widespread phytopathogenic fungus that can cause gray mold rot or Botrytis blight of many vegetables, ornamentals, fruits and even field crops throughout the world ${ }^{[18]}$. Chemical control of Botrytis is not effective and often associated with the appearance of resistant Botrytis strains ${ }^{[19]}$. Recently, biological control using natural substances has alleviated the negative impacts of chemical control, and has brought huge economic benefits ${ }^{[20]}$. 
Pathogenesis-related (PR) proteins are important in plant defense against pathogens, some PR proteins have been identified as chitinases and $\beta-1,3$-glucanases ${ }^{[21]}$. The high activities of these two proteins was potentially related to resistance to Alternaria solani in potato ${ }^{[22]}$. The synthesis of PR proteins is regulated at the transcriptional level and it has often been suggested that the collection of PR proteins may be effective in inhibiting pathogen growth and inducing systemic acquired resistance ${ }^{[23,24]}$. Originally, five main groups of PRs (PR-1 to PR-5) were characterized by both molecular and molecular-genetic techniques in tobacco, numbered in order of decreasing electrophoretic mobility ${ }^{[25]}$. Later more novel families (PR-6 to PR-16) were recognized in other plants ${ }^{[26-30]}$. Overexpression of a variety of these PR genes in transgenic plants resulted in enhanced resistance to different fungal pathogens ${ }^{[31,32]}$. Thus, their synthesis serves as a marker for disease resistance.

Paenibacillus polymyxa strain A21, which was previously isolated from a snow-covered high-altitude area of Tibet, China and characterized in our laboratory, had a high antagonistic activity against $B$. cinerea $^{[33]}$. The main purpose of this study was to identify the antifungal compounds from the strain $P$. polymyxa A21 and test their effectiveness to control gray mold diseases on tomato caused by $B$. cinerea. In addition, comparative cluster analysis of fusaricidin BGCs in three $P$. polymyxa strains and mRNA levels of three PR proteins induced by fusaricidin was undertaken.

\section{Materials and methods}

\subsection{Strains}

P. polymyxa A21 (CGMCC accession no. 10708) was cultured on King's B modified medium $(\mathrm{KBM})^{[34]}$ agar at $30^{\circ} \mathrm{C}$. The plant pathogens used in this study were a: B. cinerea, Ceratobasidium cereale, Colletotrichum capsici, Fusarium graminearum, Fusarium solani, F. oxysporum f. sp. vasinfectum, F. oxysporum f. sp. niveum, $F$. oxysporum sp. cucumerinum, $F$. oxysporum f. sp. Conglutinan, Glomerella cingulata, and Monilia laxa. These pathogens were provided by the Institute of Plant and Environment Protection, Beijing Academy of Agriculture and Forestry Sciences, and the Department of Plant Pathology, China Agricultural University.

\subsection{Antimicrobial spectrum assay of Paenibacillus poly- myxa A21}

The antimicrobial spectrum of antifungal substances produced by strain A21 was determined according to $\mathrm{Lu}$ et al. ${ }^{[35]}$ with minor modifications. Plant pathogenic fungi were cultured on potato dextrose agar ${ }^{[36]}$ slants at $25^{\circ} \mathrm{C}$ for 5-7 d. Pre-poured agar media plates were spread with
$200 \mu \mathrm{L}$ of the harvested spore suspension $\left(10^{6}\right.$ spores per milliliter). Two wells ( $7 \mathrm{~mm}$ diameter) were prepared in the agar plate, one was filled with $100 \mu \mathrm{L}$ of the antifungal substances, and the other was filled with KBM broth as the control. The plates were incubated at $25^{\circ} \mathrm{C}$ for $2 \mathrm{~d}$. The antimicrobial activity was evaluated by measuring the diameters of the round inhibition zones with calipers. Each treatment was replicated three times.

\subsection{Extraction and purification of antimicrobial compounds}

For preparation of the antimicrobial compounds of strain A21, KBM broth $(100 \mathrm{~mL})$ was inoculated with $1 \mathrm{~mL}$ of overnight culture of A21 grown in potato dextrose broth and incubated in an incubator shaker $\left(200 \mathrm{r} \cdot \mathrm{min}^{-1}, 30^{\circ} \mathrm{C}\right)$. After $3 \mathrm{~d}$, the liquid culture was centrifuged at $12000 \mathrm{~g}$ for $10 \mathrm{~min}$ and the antimicrobial compounds were extracted twice with n-butanol from the supernatant and purified with a silica gel column according to Raza et al. ${ }^{[15]}$. Different active fractions were collected and dissolved in methanol.

The antimicrobial compounds were fractioned by reversed phase high performance liquid chromatography (RP-HPLC) (C18 column, $250 \mathrm{~mm} \times 4.6 \mathrm{~mm}$, Agilent 1100 series; Agilent Technologies, Palo Alto, CA, USA) according to Deng et al. ${ }^{[37]}$. A $20 \mu \mathrm{L}$ aliquot of extract was loaded and monitored at $210 \mathrm{~nm}$ by elution with a linear biphasic gradient of $10 \%$ to $90 \%$ solvent A (water containing $0.1 \%$ trifluoroacetic acid (TFA)) and solvent $\mathrm{B}$ (acetonitrile with $0.1 \%$ TFA) in 60 min, with a flow rate of $0.5 \mathrm{~mL} \cdot \mathrm{min}^{-1}$

\subsection{Mass spectrometry}

The active HPLC fractions were analyzed by a hybrid ion trap time-of-flight mass spectrometer (Shimadzu, Kyoto, Japan) equipped with an electrospray ionization interface and connected to an LC system via a PEEK tube $(0.13 \mathrm{~mm}$ i.d.) to perform high-resolution tandem mass spectrometry under the same conditions as described for the HPLC purification, except that $10 \mu \mathrm{L}$ of sample was injected. The mass spectrometric parameters were set as below according to Sun et al. ${ }^{[38]}$ : ionization polarity, positive; nebulizing gas flow rate, $1.5 \mathrm{~L} \cdot \mathrm{min}^{-1}$; drying gas pressure, $100 \mathrm{MPa}$; detector voltage, $1.40 \mathrm{kV}$; curved desolvation line (CDL) voltage, constant level; CDL temperature, $200^{\circ} \mathrm{C}$; block heater temperature, $200^{\circ} \mathrm{C}$; interface voltage, $1.4 \mathrm{kV}$; and IT vacuum, $1.9 \times 10^{-2} \mathrm{~Pa}$. The ion accumulation time was set at $100 \mathrm{~ms}$. The collision energy of CID was set at $50 \%$. Data acquisition was in both positive and negative ion modes.

2.5 Cloning the fusaricidin biosynthetic gene clusters of Paenibacillus polymyxa A21

Genomic DNA of $P$. polymyxa A21 was prepared with a 
TIANamp Bacteria DNA Kit (Tiangen, Beijing, China). Nine pairs of specific primers for sequences of eight genes and seven intergenic regions were designed according to the sequence of the fusaricidin gene cluster of $P$. polymyxa strain SC2 (EU431181.2) and used to clone the fusaricidin BGCs from P. polymyxa A21. All the PCR products were sequenced and assembled using DNAMAN and NCBI/ BLAST database ${ }^{[39]}$. GenBank accession number for fusaricidin BGCs of P. polymyxa A21 is KT377184. Synthesis of oligonucleotide primers and DNA sequencing of PCR products were performed by Invitrogen (Beijing, China). All the primers sequences were listed in Table S1.

2.6 The control effect of antimicrobial compounds against $B$. cinerea on tomato leaves and fruits

To prepare the crude fusaricidin extract of strain A21, KBM broth $(100 \mathrm{~mL})$ was inoculated with $1 \mathrm{~mL}$ of overnight culture of A21 grown in potato dextrose broth and incubated in an incubator shaker $\left(200 \mathrm{r} \cdot \mathrm{min}^{-1}, 30^{\circ} \mathrm{C}\right)$. After $3 \mathrm{~d}$, liquid culture was centrifuged at $12000 \mathrm{~g}$ for $10 \mathrm{~min}$ and the antimicrobial compounds were extracted twice with n-butanol from the supernatant. The extract was concentrated using a rotary evaporator and the residues dissolved in $10 \mathrm{~mL}$ methanol.

The effects of antimicrobial compounds from A21 on controlling gray mold disease caused by $B$. cinerea were studied on detached tomato leaves and fruit. Healthy similar sized tomato leaves (Solanum lycopersicum cv. Jiafen No.19) were collected when the seedlings had a fully expanded fourth leaf. The detached leaves were washed with sterile water and placed in a culture dish with sterile filter paper. Pathogens were pre-cultured for 2 days on the PDA cut as a block (diameter $1 \mathrm{~cm}$ ) and placed on the leaves of tomato, and then the crude fusaricidin extract from strain A21 and chemical fungicide was applied as a spray according to the methods of Jiang et al. ${ }^{[0]}$. Harvested mature tomato fruit were surface-disinfected with $1 \%$ commercial sodium hypochlorite, and then rinsed with sterilized water, and three artificial wounds were made along the equatorial zone of the fruits. Thirtymicrolitre aliquots of $B$. cinerea suspension at $1 \times 10^{6}$ conidia per milliliter were pipetted into each wound, and the fruits, after air-drying for $2 \mathrm{~h}$ at $25^{\circ} \mathrm{C}$, and $30 \mu \mathrm{L}$ the crude fusaricidin extract and chemical fungicide were pipetted into each wound as described by Zhang et al. ${ }^{[41]}$.

Four treatments were applied: (1) 1:5 dilution of the crude fusaricidin extract of strain A21 applied before inoculation; (2) chemical fungicide Boscalid WG $1000 \times$ (BASF, Limburgerhof, Germany) at $500 \mu \mathrm{g} \cdot \mathrm{mL}^{-1}$ applied before inoculation; (3) inoculated plants with no other treatment as inoculated controls and (4) uninoculated, water-treated plants as negative controls. Fifteen tomato leaves and fruit were used for each treatment, and treatments replicated three times. The diseased leaf rate, lesion diameters of tomato leaves and fruits were surveyed and control rates were calculated according to Lu et al. ${ }^{[42]}$. The experiment was repeated three times.

\subsection{Defense gene expression analysis in tomato leaves after treatment with fusaricidin extract}

Quantitative real time PCR (qRT-PCR) was used to quantify mRNA levels of antifungal proteins of defense genes $P R-1, P R-2$ and $P R-3$ in tomato leaves inoculated with $B$. cinerea only and leaves sprayed with fusaricidin extract of strain A21 before $B$. cinerea inoculation. Total RNA was extracted using RNeasy Mini kit (Tiangen, Beijing, China) from the leaves $36 \mathrm{~h}$ after treatment, and $1 \mu \mathrm{g}$ of total RNA was used for cDNA (cDNA) synthesis as previously described ${ }^{[43]}$. Primer pairs used for qRT-PCR were designed using Primer 5 software (PREMIER Biosoft International, Silicon Valley, CA, USA). In a series of initial experiments, the performance of the designed primers (Table S1) was tested by qRT-PCR using the cDNA templates. qRT-PCR analysis was carried out using Takara SYBR ${ }^{\circledR}$ Premix Ex Taq ${ }^{\text {TM }}$ II (Tli RNaseH Plus; Takara, Beijing, China) supplemented with ROX in an ABI GeneAmp PCR System 9700. The expression of each gene was normalized to the expression of actin.

\subsection{Statistics}

All experiments were conducted in triplicate, and the average values are reported. Key results were repeated three times to establish their validity, determined using analysis of variance (ANOVA), and expressed by their mean \pm standard deviation. Variations between treatment means were compared by one-way ANOVA

\section{Results}

\subsection{Antimicrobial activity assay}

The antifungal substances produced by strain A21 exhibited inhibitory effects in vitro on all the 12 plant pathogenic fungi tested, although to different degrees (Table 1). The inhibition of B. cinerea and Glomerella cingulate in particular were the most dramatic, with diameters of inhibition zones of up to 3.4 and $3.2 \mathrm{~cm}$, respectively. The KBM control had no activity against any of the fungi.

\subsection{Characteristics of antifungal compounds}

After the final purification by RP-HPLC, antifungal activity was observed in one major distinct peak obtained at an elution time of $24.5 \mathrm{~min}$ (Fig. 1a). This fraction was separately collected using the same RP-HPLC program, and prepared for structural analysis. The chemical structures of these purified fractions were elucidated by 
Table 1 Antifungal activities of the crude extract of Paenibacillus polymyxa strain A21

\begin{tabular}{lc}
\hline Fungal pathogens & Average diameter of inhibition zone/cm \\
\hline Botrytis cinerea & $3.43 \pm 0.08$ \\
Glomerella cingulata & $3.16 \pm 0.14$ \\
Botrytis cinerea & $2.66 \pm 0.15$ \\
Fusarium oxysporum f. sp. niveum & $2.62 \pm 0.10$ \\
F. graminearum & $2.34 \pm 0.07$ \\
Monilia cinerea & $1.93 \pm 0.09$ \\
Vermicularia capsici & $1.93 \pm 0.13$ \\
F. oxysporum f. sp. vasinfectum & $1.65 \pm 0.06$ \\
Rhizoctonia cerealis & $1.62 \pm 0.03$ \\
F.oxysporum f. sp. cucumerinum & $1.62 \pm 0.12$ \\
F. solani & $1.16 \pm 0.11$ \\
F. oxysporum f. sp. conglutinan & $1.12 \pm 0.04$ \\
\hline
\end{tabular}

(a)

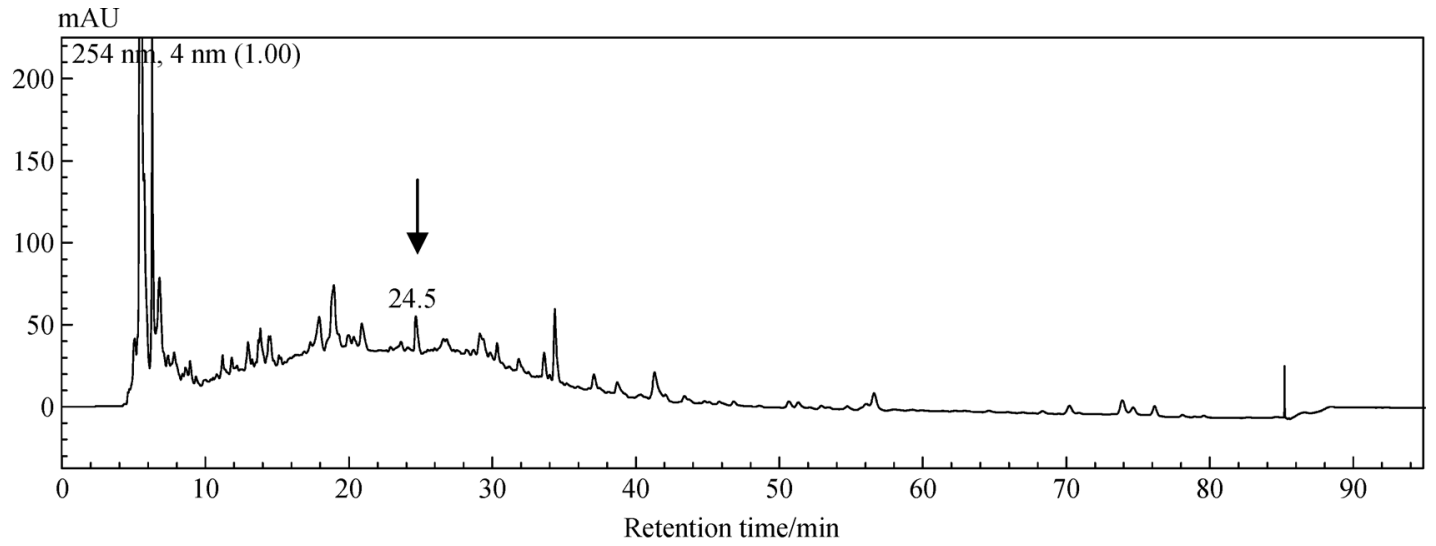

(b)

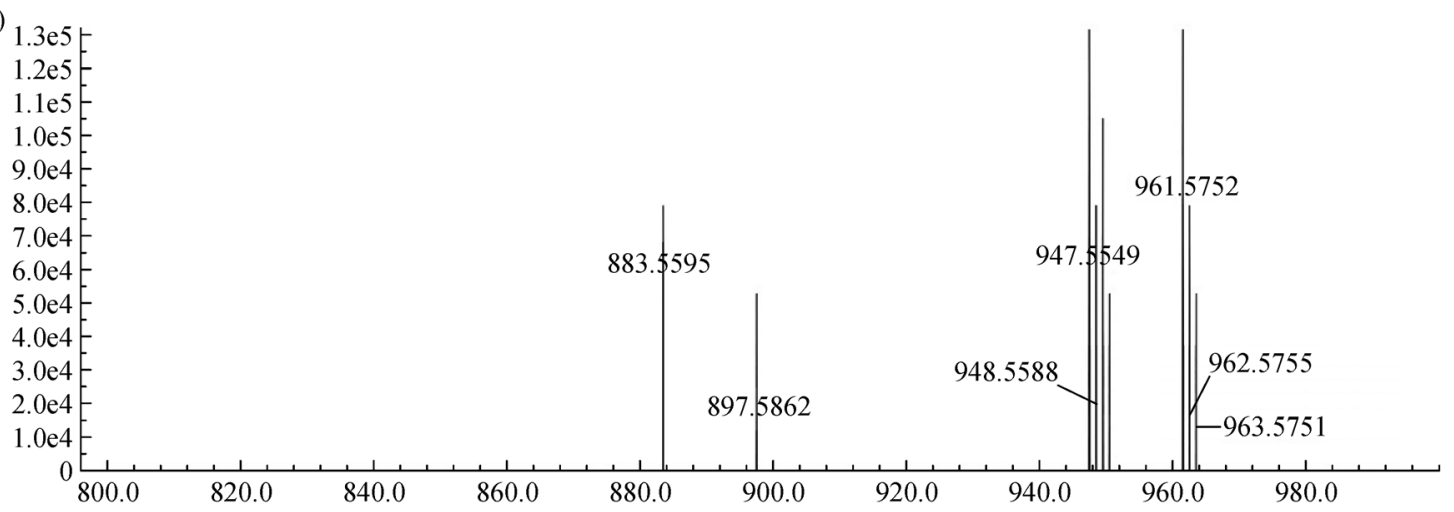

Fig. 1 RP-HPLC peak profile of antifungal compounds from Paenibacillus polymyxa A21. (a) Antifungal activity was found in the peak eluted at $24.5 \mathrm{~min}$, as indicated by the arrow; (b) mass spectroscopic analysis of antifungal compounds produced by $P$. polymyxa A21. Compounds from peaks with molecular weights 947.56 , 961.58, 883.56 and $897.59 \mathrm{Da}(\mathrm{M}+\mathrm{H})^{+}$showed antifungal activity. Mass spectroscopy was done in both positive and negative ion modes.

LC-DAD-IT-TOF-MS. The LC-MS analysis showed that this peak was comprised of four compounds of molecular weights $947.55,961.58,883.56$ and $897.59 \mathrm{Da}(\mathrm{M}+\mathrm{H})^{+}$ (Fig. 1b), corresponding to the molecular formula of $\mathrm{C}_{45} \mathrm{H}_{74} \mathrm{~N}_{10} \mathrm{O}_{12}, \mathrm{C}_{46} \mathrm{H}_{76} \mathrm{~N}_{10} \mathrm{O}_{12}, \mathrm{C}_{41} \mathrm{H}_{74} \mathrm{~N}_{10} \mathrm{O}_{11}$, and $\mathrm{C}_{42} \mathrm{H}_{76} \mathrm{~N}_{10} \mathrm{O}_{11}$, respectively.
Comparing the mass data with those obtained in previous studies ${ }^{[13]}$, the series of molecular ions in the present study are likely to represent LI-F antibiotic homologs. By comparison with fusaricidins studied previously ${ }^{[13,15,37,44]}$, the compounds with molecular weights of $947.55,961.58,883.56$ and $897.59 \mathrm{Da}$ 
$(\mathrm{M}+\mathrm{H})^{+}$were designated as fusaricidin $\mathrm{C}, \mathrm{D}, \mathrm{A}$ and $\mathrm{B}$, respectively.

3.3 Cloning and comparative cluster analysis of fusaricidin biosynthetic gene clusters

The fusaricidin BGCs from $P$. polymyxa A21 were cloned using nine pairs of specific primers (Table S1) designed according to the sequences of fusaricidin BGCs in P. polymyxa PKB1 and SC2. The fusaricidin BGCs of $P$. polymyxa A21 contains eight open reading frames (ORFs) putatively involved in fusaricidin biosynthesis, spanning 31824 bp of DNA sequence (Fig. 2a). To perform the comparative cluster analysis, the complete sequences of the fusaricidin BGCs of $P$. polymyxa A21, $P$. polymyxa PKB1 (EF451155.3) and P. polymyxa SC2 (EU431181.2) were aligned using the MAUVE program ${ }^{[45]}$. To facilitate a comparative analysis with the fusaricidin BGCs of $P$. polymyxa $\mathrm{PKB} 1$ (pfur cluster) ${ }^{[14]}$ and $P$. polymyxa $\mathrm{SC} 2$ (sfur cluster), we named the cluster from $P$. polymyxa A21 the afur cluster.

The comparative analysis revealed that the fusaricidin BGCs of strains A21 and SC2 share the same architecture and orthologous genes, and no gene insertion or rearrangement was found in the BGCs (Fig. 2b). All genes from the afur and sfur clusters had a higher sequence identity $(\geqslant 96 \%)$ than those from the afur and pfur clusters $(\geqslant 91 \%)$. The afur cluster contains all of the ORFs identified in $P$. polymyxa SC2, including the $23.7 \mathrm{~kb}$ non-ribosomal peptide synthetase gene fus $A$ and seven other genes (fusB, fusC, fusD, fusE, fusF, fus $G$ and fusTE) involved in fusaricidin production. All of the putative proteins of the fusaricidin BGCs of $P$. polymyxa A21 showed significantly high similarity (most $\geqslant 96 \%)$ to their counterparts in $P$. polymyxa $\mathrm{SC} 2$, suggesting that the two strains may share the same metabolic regulatory mechanisms in the fusaricidin biosynthetic pathway.

The gene fusTE in strain PKB1, a major strain-specific region, did not have an orthologous gene in fusaricidin BGCs of strains A21 and SC2, although a 399 bp ORF downstream of fus $A$ and oriented in the opposite orientation was annotated as the fusTE gene in strain SC2. No similarity was found between the nucleotide and amino acid sequences of the unknown ORFs in strain A21 and the fusTE gene in PKB1, but the unknown ORFs in strains A21 and annotated fusTE gene in SC2 had complete identical sequences. Based on the BlastP analysis, sequences of strains $\mathrm{A} 21$ and $\mathrm{SC} 2$ did not have the conserved GxSxG motif of thioesterases, which was found in the fusTE sequence of strain PKB1. These sequence difference between afur and pfur clusters in A21 and PKB1 indicated the evolution of antibiotic BGCs.

(a)

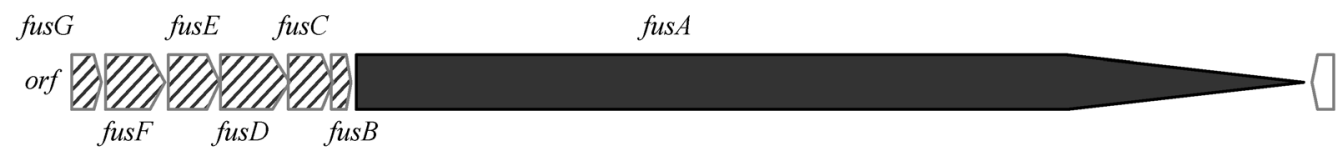

(b)
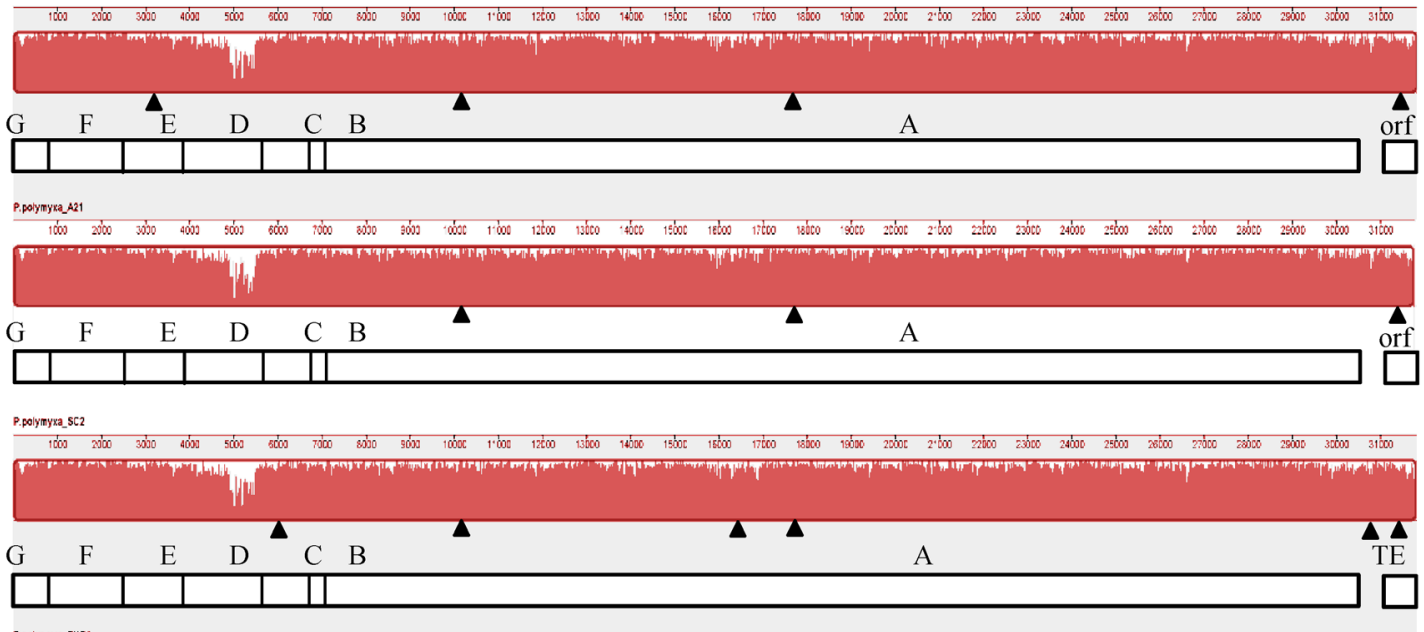

Fig. 2 Analysis of fusaricidin biosynthetic gene clusters (BCGs). (a) BGCs of Paenibacillus polymyxa A21; (b) comparison of the fusaricidin BGCs of afur, sfur and pfur clusters using the MAUVE program. The horizontal panels depict the afur (upper), sfur (middle) and pfur (lower) clusters. Mean sequence similarities are proportional to the heights of the red bars, and the respective scales show the sequence coordinates in base pairs. Regions with low similarities and strain-specific regions are marked with black triangles. Arrows indicate deduced transcriptional units. 
3.4 Effect of fusaricidin extract on Botrytis cinerea on tomato leaves and fruit

After $5 \mathrm{~d}$, the tomato leaves inoculated with $B$. cinerea alone were all severely diseased (disease incidence 100\%), with the average lesion diameter of $30.2 \pm 2.3 \mathrm{~mm}$. Leaves treated with fusaricidin extract of strain A21 before $B$. cinerea inoculation had significantly reduced gray mold disease incidence $(35 \%)$ and lesion size $(7.7 \mathrm{~mm})$, which was comparable to those plants treated with Boscalid WG (Table 2). Similarly, fusaricidin extract of strain A21 also gave excellent suppression of gray mold infection on tomato fruit. Compared with the inoculated controls, which had a $100 \%$ disease incidence and a lesion diameter of $37.6 \pm 2.5 \mathrm{~mm}$, tomato fruit treated with fusaricidin extract of strain A21 did not show any gray mold symptoms 7 and $10 \mathrm{~d}$ after inoculation, the same as for fruit treated with $500 \mu \mathrm{g} \cdot \mathrm{mL}^{-1}$ Boscalid and the negative controls. These results demonstrated that fusaricidin extract from strain A21 significantly inhibited the development of the gray mold diseases caused by B. cinerea on tomato leaves and fruit, and that disease control was more effective on fruit.

3.5 Effect of fusaricidin extract from A21 on defenserelated gene expression in tomato leaves

qRT-PCR was carried out to determine if defense genes, $P R-1, P R-2$ and $P R-3$, were activated in leaves of tomato treated with fusaricidin extract. Gene expression analysis performed at $36 \mathrm{~h}$ after treatment showed that all the defense genes were induced in tomato leaves pretreated with fusaricidin extract. Fusaricidin treatment resulted in significantly higher expression of $P R-2$ mRNA in comparison to the inoculated control (Fig. 3). The response of $P R-1$ to fusaricidin was not as strong, but all the PRs detected displayed higher expression than the controls. These results indicated that the fusaricidin induced disease resistance in tomato via the activation of defense-related genes.

\section{Discussion}

Paenibacillus polymyxa strain A21 isolated from a snowcovered high-altitude area of Tibet, China, exhibited a high antagonistic activity against $B$. cinerea $^{[33]}$. In this study, the antimicrobial compounds of strain A21 extracted with n-butanol showed significant antagonistic activity against 12 fungal pathogens. After purification with RP-HPLC, the active HPLC fractions were analyzed by LC-DAD-ITTOF-MS. The molecular weights and structural data revealed that the antimicrobial compounds belonged to the fusaricidin-type antibiotic. Several strains of $P$. polymyxa have been reported to produce fusaricidin, and various analogs of fusaricidins have been isolated and characterized, including LI-F03, LIF04, LI-F05, LI-F06,

Table 2 Antagonistic activity of the fusaricidin extract from Paenibacillus polymyxa strain A21 against Botrytis cinerea infection of tomato leaves

\begin{tabular}{lcc}
\hline Treatment & Disease incidence $/ \%$ & Average diameter of lesion $/ \mathrm{mm}^{2}$ \\
\hline Fusaricidin extract from A21 & $35 \pm 2.4 \mathrm{~b}$ & $7.7 \pm 0.4 \mathrm{~b}$ \\
Botrytis cinerea inoculated control & $100 \pm 0.0 \mathrm{a}$ & $30.2 \pm 2.3 \mathrm{a}$ \\
Boscalid & $32 \pm 1.9 \mathrm{~b}$ & $6.9 \pm 0.2 \mathrm{~b}$ \\
Blank control & $0 \pm 0.0 \mathrm{c}$ & $0 \pm 0.0 \mathrm{c}$ \\
\hline
\end{tabular}

Note: The results are the mean of two independent experiments \pm SD. Values followed by same letters are not statistically different by Duncan's multiple range test $(P<0.05)$

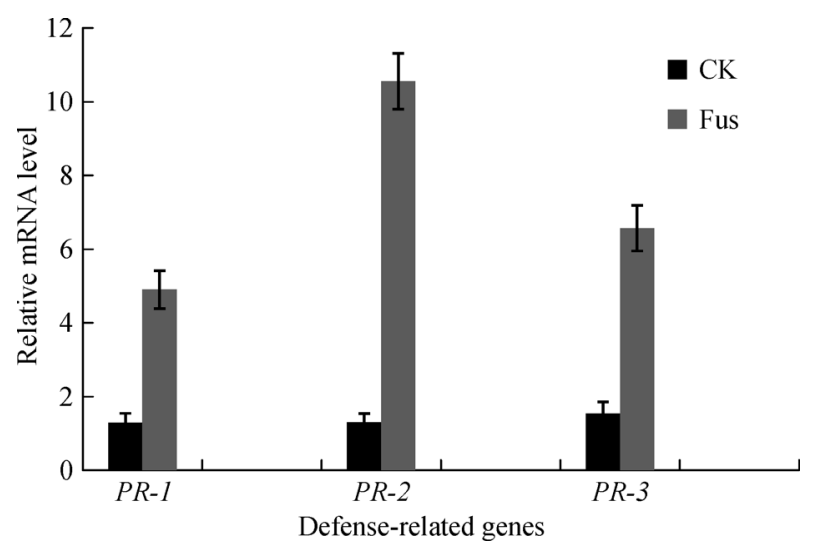

Fig. 3 Relative gene expression of defense-related genes $P R-1, P R-2$ and $P R-3$ in tomato leaves treated with fusaricidin analyzed by qRT-PCR (Fus, fusaricidin treatment before inoculation with B. cinerea; CK, inoculated control). $Y$-axes scale is relative expression level. Error bars indicate \pm SD. 
LI-F07 and LI-F08 ${ }^{[46]}$, as well as fusaricidin A, B, C and $\mathrm{D}^{[37]}$. Comparison of our results with the previous literature revealed that the fusaricidin-type antibiotics produced by $P$. polymyxa A21 were fusaricidin A, B, C and D. Importantly, treatment with the fusaricidin extract from A21 greatly reduced the gray mold disease incidence and severity on both tomato leaves and fruit, indicating that strain A21 is a potential biocontrol agent against the gray mold diseases caused by $B$. cinerea on tomato.

Comparative cluster analysis of Paenibacillus spp. and the fusaricidin-types in different strains indicated no relationship between the similarity of gene clusters and fusaricidin-types, and the sequence differences between the fusaricidin clusters of Paenibacillus spp. reveals the occurrence of the evolution of antibiotic BGCs. Thus, we speculate that all types are produced by the Paenibacillus spp. with the amount of different fusaricidins varying with fermentation conditions and strain. Variation in reports of production of certain types of fusaricidins might be explained if some were present at concentrations too low to detect.

The fact that the unknown ORF in A21 had no sequence similarity with the fusTE gene in PKB1 indicates that fusTE is not needed for fusaricidin synthesis. Previously, the fusTE gene product has been assigned as the $3^{\prime}$ boundary of the fusaricidin gene cluster according its greatest similarity to proteins of the $\alpha / \beta$ hydrolase fold family ( $45 \%$ identity), and it was presumed to catalyze the hydrolysis of acyl or aminoacyl groups inappropriately attached to the 40-PP cofactors of fusaricidin synthetase or participate in the transferring of the fatty acid of fusaricidin ${ }^{[14]}$. However, there have been no gene disruption studies conducted to confirm its involvement, so its exact function remains unclear. Thus, determination of the function of gene fusTE in fusaricidin biosynthesis in Paenibacillus spp. is still needed.

Many studies have shown that biotic and abiotic agents can protect plants against invading pathogens either directly by antagonistic mechanisms or indirectly through induced systemic resistance in host plants. Fusaricidin can induce protection from phytophthora leaf blight of red pepper and soft rot of tobacco through systemic resistance ${ }^{[44]}$. In this study, we have shown that the transcriptional levels of three defense genes were induced by fusaricidin, indicating that fusaricidin induced disease resistance in tomato via the activation of a series of PRs to protect the plants from $B$. cinerea infection. In fact, the gray mold on tomato leaf and fruit was suppressed by spraying and injection of fusaricidin, implying that the mechanism of disease suppression is through systemic or/ and direct antagonism. So, further work will focus on to the separation of individual fusaricidins from strain A21, and testing their ability to protect the tomato from gray mold by soil drench and foliar spray and to induce defense genes in tomato.

\section{Conclusions}

Paenibacillus polymyxa A21 isolated from a snow-covered high-altitude area of Tibet, China, exhibited a high antagonistic activity against $B$. cinerea. Four kinds of antifungal compounds from an extract of strain A21 with molecular masses of 883.56, 897.59, 947.55, and $961.58 \mathrm{Da}$ were characterized as the members of fusaricidin-type of antibiotics according to LC-MS analysis. Moreover, treatment with the fusaricidin extract from A21 greatly reduced the gray mold disease incidence and severity on both tomato leaves and fruit, indicating that strain A21 is a potential biocontrol agent against the gray mold diseases caused by $B$. cinerea on tomato. Comparative cluster analysis of Paenibacillus spp. and the fusaricidin-types in different strains indicated no relationship between the similarity of gene clusters and fusaricidin-types. The gene fusTE, the 3' boundary of the fusaricidin BGC in strain PKB1, was not present in fusaricidin $\mathrm{BGC}$ of $\mathrm{A} 21$, indicating that fusTE is not necessary for fusaricidin synthesis. The mRNA levels for three pathogenesis-related proteins (PRs) revealed that treatment of tomato leaves with fusaricidin extract induced the expression of PR genes to different levels, suggesting that one reason for the reduction of gray mold infection by fusaricidin is induction of PR proteins, which lead to increased resistance to pathogens.

Supplementary materials The online version of this article at https://doi. org/10.15302/J-FASE-2017166 contains supplementary material (Tables S1).

Acknowledgements This work was supported by the Scientific and Technological Innovation Capacity Construction Special Funds, Beijing Academy of Agriculture and Forestry (KJCX20170410), the Science and Technology Innovation Fund from the Beijing Academy of Agriculture and Forestry Sciences (QNJJ201519), and Beijing Key Laboratory of Environment Friendly Management on Fruit Diseases and Pests in North China (BZ0432).

Compliance with ethics guidelines Weicheng Liu, Xiaoli Wu, Xuelian Bai, Hong Zhang, Dan Dong, Taotao Zhang, and Huiling Wu declare that they have no conflicts of interest or financial conflicts to disclose.

This article does not contain any studies with human or animal subjects performed by any of the authors.

\section{References}

1. Ash C, Priest F G, Collins M D. Molecular identification of rRNA group 3 Bacilli (Ash, Farrow, Wallbanks and Collins) using PCR probe test. Proposal for the creation of a new genus Paenibacillus. Antonie van Leeuwenhoek, 1993, 64(3-4): 253-260

2. Khan Z, Kim S G, Jeon Y H, Khan H U, Son S H, Kim Y H. A plant growth promoting rhizobacterium, Paenibacillus polymyxa strain GBR-1, suppresses root-knot nematode. Bioresource Technology, 2008, 99(8): 3016-3023

3. Kharbanda P D, Yang J, Beatty P, Jensen S, Tewari J P. Biocontrol 
of Leptospheria maculansand other pathogens of canola with Paenibacillus polymyxa PKB1. In: Proceeding of 10th International Rapeseed Congress, Canberra, 1999

4. Ryu C M, Kim J, Choi O, Kim S H, Park C S. Improvement of biological control capacity of Paenibacillus polymyxa E681 by seed pelleting on sesame. Biological Control, 2006, 39(3): 282-289

5. Ito M, Koyama Y. Jokipeptin, a new peptide antibiotic. I. Isolation, physico-chemical and biological characteristics. Journal of Antibiotics, 1972, 25(5): 304-308

6. Shoji J, Kato T, Hinoo H. The structure of polymyxin T1. (Studies on antibiotics from the genus Bacillus. XXII). Journal of Antibiotics, 1977, 30(12): 1042-1048

7. Pichard B, Larue J P, Thouvenot D. Gavaserin and saltavalin, new peptide antibiotics produced by Bacillus polymyxa. FEMS Microbiology Letters, 1995, 133(3): 215-218

8. Kaye D. Current use for old antibacterial agents: polymyxins, rifampin, and aminoglycosides. Infectious Disease Clinics of North America, 2004, 18(3): 669-689

9. Nakajima N, Chihara S, Koyama Y. A new antibiotic, gatavalin. I. Isolation and characterization. Journal of Antibiotics, 1972, 25(4): 243-247

10. Kajimura Y, Kaneda M. Fusaricidin B,C and D, new depsipeptide antibiotics produced by Bacillus polymyxa KT-8, isolation, structure elucidation and biological activity. Journal of Antibiotics, 1997, 50 (3): 220-228

11. Kajimura Y, Kaneda M. Fusaricidin A, a new depsipeptide antibiotic produced by Bacillus polymyxa KT-8. Taxonomy, fermentation, isolation, structure elucidation and biological activity. Journal of Antibiotics, 1996, 49(2): 129-135

12. Beatty P H, Jensen S E. Paenibacillus polymyxa produces fusaricidin-type antifungal antibiotics active against Leptosphaeria maculans, the causative agent of blackleg disease of canola. Canadian Journal of Microbiology, 2002, 48(2): 159-169

13. Han J W, Kim E Y, Lee J M, Kim Y S, Bang E, Kim B S. Sitedirected modification of the adenylation domain of the fusaricidin nonribosomal peptide synthetase for enhanced production of fusaricidin analogs. Biotechnology Letters, 2012, 34(7): 13271334

14. Li J, Jensen S E. Nonribosomal biosynthesis of fusaricidins by Paenibacillus polymyxa PKB1 involves direct activation of a Damino acid. Chemistry \& Biology, 2008, 15(2): 118-127

15. Raza W, Yang X M, Wu H S, Wang Y, Xu Y C, Shen Q R. Isolation and characterisation of fusaricidin-type compound-producing strain of Paenibacillus polymyxa SQR-21 active against Fusarium oxysporum f. sp. nevium. European Journal of Plant Pathology, 2009, 125(3): 471-483

16. Nomura T, Kuroda J, Fukai T, Konishi M, Uno J, Kurusu K. LI-F antibiotics, a family of antifungal cyclic depsipeptides produced by Bacillus polymyxa L-1129. Heterocycles, 2000, 53(7): 1533-1549

17. Kurusu K, Ohba K, Arai T, Fukushima K. New peptide antibiotics LI-F03, F04, F05, F07, and F08, produced by Bacillus polymyxa. I. Isolation and characterization. Journal of Antibiotics, 1987, 40(11): $1506-1514$

18. Alfonso C, Raposo R, Melgarejo P. Genetic diversity in Botrytis cinerea populations on vegetable crops in greenhouses in southeastern Spain. Plant Pathology, 2000, 49(2): 243-251
19. Rosslenbroich H J, Stuebler D. Botrytis cinerea-history of chemical control and novel fungicides for its management. Crop Protection, 2000, 19(8): 557-561

20. Park J K, Kim J D, Park Y I, Kim S K. Purification and characterization of a 1,3- $\beta$-d-glucanase from Streptomyces torulosus PCPOK-0324. Carbohydrate Polymers, 2012, 87(2): 1641-1648

21. Fukuda Y, Shinshi H. Characterization of a novel cis-acting element that is responsive to a fungal elicitor in the promoter of a tobacco class I chitinase gene. Plant Molecular Biology, 1994, 24(3): 485493

22. Shahbazi H, Aminian H, Sahebani N, Halterman D A. Activity of $\beta$ 1, 3-glucanase and $\beta$-1,4-glucanase in two potato cultivars following challenge by the fungal pathogen Alternaria solani. Phytoparasitica, 2011, 39(5): 455-460

23. Kombrink E, Somssich I E. Pathogenesis-related proteins and plant defense. In: Carroll G, Tudzynski P, eds. The Mycota V, Part A. Plant Relationships. Berlin: Springer Verlag, 1997: 107-128

24. Ryals J A, Neuenschwander U H, Willits M G, Molina A, Steiner H Y, Hunt M D. Systemic acquired resistance. Plant Cell, 1996, 8(10): 1809-1819

25. Bol J F, Linthorst H J M, Cornelissen B J C. Plant pathogenesisrelated proteins induced by virus infection. Annual Review of Phytopathology, 1990, 28(1): 113-138

26. van Loon L C, Pierpoint W S, Boller T, Conejero V. Recommendations for naming plant pathogenesis-related proteins. Plant Molecular Biology Reporter, 1994, 12(3): 245-264

27. van Loon L C, van Strien E A. The families of pathogenesis-related and comparative analysis of PR-1 type proteins. Physiological and Molecular Plant Pathology, 1999, 55(2): 85-97

28. Park C J, Kim K J, Shin R, Park J M, Shin Y C, Paek K H. Pathogenesis-related protein 10 isolated from hot pepper functions as a ribonuclease in an antiviral pathway. Plant Journal, 2004, 37 (2): 186-198

29. Park C J, An J M, Shin Y C, Kim K J, Lee B J, Paek K H. Molecular characterization of pepper germin-like protein as the novel PR-16 family of pathogenesis-related proteins isolated during the resistance response to viral and bacterial infection. Planta, 2004, 219(5): 797-806

30. Edreva A. Pathogenesis-related proteins: research progress in the last 15 years. General and Applied Plant Physiology, 2005, 31(1-2): $105-124$

31. Klessig D F, Malamy J. The salicylic acid signal in plants. Plant Molecular Biology, 1994, 26(5): 1439-1458

32. Ryals J, Uknes S, Ward E. Systemic acquired resistance. Plant Physiology, 1994, 104(4): 1109-1112

33. Li J J, Liu W C, Luo L J, Dong D, Liu T, Zhang T T, Lu C G, Liu D W, Zhang D P, Wu H L. Expression of Paenibacillus polymyxa $\beta$ 1,3-1,4-glucanase in Streptomyces lydicus A01 improves its biocontrol effect against Botrytis cinerea. Biological Control, 2015, 90(12): 141-147

34. Atlas R M. Handbook of media for environmental microbiology. Physiological and Molecular Plant Pathology, 1995, 34: 3-12

35. Lu C G, Liu W C, Qiu J Y, Wang H M, Liu T, De Liu W. Identification of an antifungal metabolite produced by a potential biocontrol Actinomyces strain A01. Brazilian Journal of Microbiology, 2008, 39(4): 701-707 
36. Zhou D Q. Microbiology lab manual. Shanghai: Shanghai Scientific and Technical Publishers, 1996

37. Deng Y, Lu Z, Lu F, Zhang C, Wang Y, Zhao H, Bie X. Identification of LI-F type antibiotics and di-n-butyl phthalate produced by Paenibacillus polymyxa. Journal of Microbiological Methods, 2011, 85(3): 175-182

38. Sun J, Song Y L, Zhang J, Huang Z, Huo H X, Zheng J, Zhang Q, Zhao Y F, Li J, Tu P F. Characterization and quantitative analysis of phenylpropanoid amides in eggplant (Solanum melongena L.) by high performance liquid chromatography coupled with diode array detection and hybrid ion trap time-of-flight mass spectrometry. Journal of Agricultural and Food Chemistry, 2015, 63(13): 34263436

39. Zheng J J, Lin Q, He P X, He X S. Morphological identification and ITS phylogenetic analysis of Hypholoma tuberosum, a new record of Hypholoma in china. Journal of Fungal Research, 2011, 9(1): 58 (in Chinese)

40. Jiang M L, Zhao R, Hu X J, Wan X, Zhang Y B. Antifungal spectrum and bio-control effect of Bacillus subtilis Tu-100 on pathogenic fungi. Chinese Journal of Oil Crop Sciences, 2009, 31 (3): 355-358 (in Chinese)

41. Zhang D P, Lu C G, Zhang T T, Spadaro D, Liu D W, Liu W C.
Candida pruni sp. nov. is a new yeast species with antagonistic potential against brown rot of peaches. Archives of Microbiology, 2014, 196(7): 525-530

42. Lu C G, Zhang D P, Liu T, Dong H P, Wang J L, Liu W C. Detection of the genes encoding lipopeptide antibiotics and biocontrol activity of Bacillus amyloliquefaciens MH71. Plant Protection, 2015, 41(3): 12-18 (in Chinese)

43. Yue Y, Zhang M, Zhang J, Tian X, Duan L, Li Z. Overexpression of the AtLOS5 gene increased abscisic acid level and drought tolerance in transgenic cotton. Journal of Experimental Botany, 2012, 63(10): 3741-3748

44. Lee S H, Cho Y E, Park S H, Balaraju K, Park J W, Lee S W, Park K. An antibiotic fusaricidin: a cyclic depsipeptide from Paenibacillus polymyxa E681 induces systemic resistance against Phytophthora blight of red-pepper. Phytoparasitica, 2013, 41(1): 49-58

45. Darling A C, Mau B, Blattner F R, Perna N T. Mauve: multiple alignment of conserved genomic sequence with rearrangements. Genome Research, 2004, 14(7): 1394-1403

46. Choi S K, Park S Y, Kim R, Lee C H, Kim J F, Park S H. Identification and functional analysis of the fusaricidin biosynthetic gene of Paenibacillus polymyxa E681. Biochemical and Biophysical Research Communications, 2008, 365(1): 89-95 